\title{
Pharmacological inhibition of EZH2 by GSK126 decreases atherosclerosis by modulating foam cell formation and monocyte adhesion in apolipoprotein E-deficient mice
}

\author{
XIANJING WEI, YING ZHANG, LIANNA XIE, KAIJUN WANG and XIAOQING WANG \\ Department of Cardiology, Affiliated Zhongshan Hospital of Dalian University, Dalian, Liaoning 116023, P.R. China
}

Received November 11, 2019; Accepted December 1, 2020

DOI: $10.3892 / \mathrm{etm} .2021 .10273$

\begin{abstract}
Histone modifications play an important role in the occurrence and development of atherosclerosis in human and atherosclerosis-prone mice. Histone methylation in macrophages, monocytes and endothelial cells markedly influence the progression of atherosclerosis. However, it remains unclear whether treatment with a histone methyltransferase enhancer of zeste homolog 2 (EZH2) inhibitor may suppress atherosclerosis. The present study aimed to determine the effects of the EZH2 inhibitor, GSK126, on the suppression and regression of atherosclerosis in apolipoprotein E-deficient mouse models. In vitro, it was found that pharmacological inhibition of EZH2 by GSK126 markedly reduced lipid transportation and monocyte adhesion during atherogenesis, predominantly through increasing the expression levels of ATP-binding cassette transporter A1 and suppressing vascular cell adhesion molecule 1 in human THP-1 cells. In vivo, it was found that atherosclerotic plaques in GSK126-treated mice were significantly decreased when comparing with the vehicle-treated animals. These results indicated that the GSK126 has the ability to attenuate the progression of atherosclerosis by reducing macrophage foam cell formation and monocyte adhesion in cell and mouse models. In conclusion, the present study provided new insights into the molecular mechanism behind the action of GSK126 and suggested its therapeutic potential for the treatment of atherosclerosis.
\end{abstract}

\section{Introduction}

Atherosclerosis is a chronic, multistep inflammatory disease characterized by cholesterol accumulation in the arterial walls $(1,2)$. The disease starts with the recruitment of macrophages and the formation of foam cells, ending with

Correspondence to: $\mathrm{Dr}$ Xiaoqing Wang, Department of Cardiology, Affiliated Zhongshan Hospital of Dalian University, 6 Jiefang Street, Dalian, Liaoning 116023, P.R. China

E-mail: wangxq_dmu@hotmail.com

Key words: EZH2, atherosclerosis, GSK126, foam cell formation, H3K27 methylation myocardial infarction, ischemic stroke or gangrene (3-10). Currently, atherosclerosis, especially coronary artery disease, remains to be a leading cause of morbidity and mortality in both developed and some developing countries $(3,4)$. However, drugs for the treatment of atherosclerosis are still under development though breakthroughs in atherosclerosis treatment could be imminent (11).

Epigenetic regulation of transcription refers to modifications in gene expression without changing the DNA sequence $(11,12)$. Additionally, epigenetic regulations are increasingly being associated with atherosclerosis in human and animal models, and are of interest from both therapeutic and biomarker perspectives (11-14). Previous studies have reported that apolipoprotein E-deficient $\left(\right.$ ApoE $\left.^{-/-}\right)$mice fed with a high-fat diet have aberrant epigenetic changes, including decreased global methylation in aorta tissue samples and peripheral blood monocytes, as well as special gene hypermethylation in atherosclerotic plaques and lesion-predisposed regions (15-18). Generally, histone modifications include methylation, acetylation, ubiquitination, phosphorylation, glycosylation and sumoylation are key epigenetic markers that play critical roles in the modification of gene expression and have been identified to play an important role in the development of atherosclerosis (19-21). Histone methylation and acetylation in macrophages and vascular endothelial cells have been reported to markedly influence the progression of atherosclerosis (21).

Histone methyltransferase enhancer of zeste homolog 2 (EZH2) is a histone methyltransferase and is the catalytic subunit of the polycomb repressive complex-2 (PRC2)/EED-EZH2 protein complex, which can catalyze trimethylation of histone $\mathrm{H} 3$ at Lys 27 (H3K27me3) to dramatically regulate gene expression through epigenetic machinery. Previous studies have found evidence that EZH2-induced downregulation of ATP binding cassette subfamily A member 1 (ABCA1) and insulin like growth factor binding protein 5 (IGFBP5) gene expression levels $(22,23)$, which promotes lipid accumulation in cells and the development of atherosclerosis. Therefore, inhibition of the histone methyltransferase EZH2 may have considerable potential for suppressing atherogenesis. GSK126 is a small-molecule inhibitor of both wild-type and mutant EZH2 methyltransferase, that has been tested in a phase $1 / 2$ dose escalation study to investigate its safety, pharmacokinetics, pharmacodynamics and clinical activity in 
patients with relapsed or refractory diffuse large B cell and transformed follicular lymphoma (24). To determine the efficacy of GSK126 for suppressing atherogenesis, the effects and mechanisms of EZH2 inhibition were investigated in vitro and in vivo in the present study.

\section{Materials and methods}

Chemicals and antibodies. The EZH2 methyltransferase inhibitor GSK126 (cat. no. S7061) was purchased from Selleck Chemicals and was dissolved in DMSO to obtain a stock solution $(100 \mu \mathrm{g} / \mathrm{ml})$ and stored at $-80^{\circ} \mathrm{C}$. The following antibodies were used in the present study: Mouse monoclonal anti-H3K27me3 (cat.no.ab6147), rabbit polyclonal anti-ABCA1 (cat. no. ab7360), rabbit monoclonal anti-vascular cell adhesion molecule 1 (VCAM1; for immunohistochemistry; cat. no. ab134047) and rabbit polyclonal anti-lectin-like oxidized low-density lipoprotein (Ox-LDL) receptor 1 (LOX-1; cat. no. ab60178) were purchased from Abcam. Rabbit monoclonal anti-VCAM1 (cat. no. PA5-102452; for immunohistochemistry) was purchased from Invitrogen. Rabbit monoclonal anti-EZH2 (cat. no. 5246), rabbit monoclonal anti- $\beta$-actin (cat. no. 8457), rabbit monoclonal anti-VCAM1 (cat. no. 13662; for western blot), rabbit monoclonal anti-intercellular adhesion molecule 1 (ICAM1; cat. no. 4915) and rabbit monoclonal anti-histone H3 (cat. no. 4499) were purchased from Cell Signaling Technology, Inc.

Cell culture. THP-1 and HUVEC cells were purchased from the American type culture collection (ATCC). THP-1 and HUVEC cells were cultured in RPMI-1640 medium (Gibco; Thermo Fisher Scientific, Inc.) supplemented with 10\% FBS (Gibco; Thermo Fisher Scientific, Inc.) and $100 \mathrm{U} / \mathrm{ml}$ penicillin-streptomycin (Gibco; Thermo Fisher Scientific, Inc.) at $37^{\circ} \mathrm{C}$ in an atmosphere containing $5 \% \mathrm{CO}_{2}$. For foam cell transformation, THP-1 cells were treated with phorbol-12-myristate-13-acetate (PMA, $100 \mathrm{nmol} / \mathrm{l}$; STEMCELL Technologies, Inc.; cat. no. 74044) for $48 \mathrm{~h}$ at $37^{\circ} \mathrm{C}$ in an atmosphere containing $5 \% \mathrm{CO}_{2}$. The medium was then replaced with a serum-free medium containing Ox-LDL (50 $\mu \mathrm{g} / \mathrm{ml}$; Thermo Fisher Scientific, Inc.; cat. no. L34357) for $48 \mathrm{~h}$ at $37^{\circ} \mathrm{C}$ in an atmosphere containing $5 \% \mathrm{CO}_{2}$ to transform THP-1 cells to foam cells.

Monocyte-endothelial cell adhesion assay. Following stimulation by Ox-LDL for $24 \mathrm{~h}$, THP-1 cells were collected by centrifugation and re-suspended in culture medium plus 2', 7 '-bis(2-carboxyethyl)-5-(and-6)-carboxyfluorescein (BCECF-AM; Beyotime Institute of Biotechnology). After incubation for an hour at $37^{\circ} \mathrm{C}$, fluorescently labeled THP-1 cells were collected by centrifugation and plated in 96 -well plates pre-seeded with HUVECs. After incubation for an hour, the plates were washed with PBS for 3 times. Images of fluorescently stained cells were captured with a fluorescence microscope (Olympus Corporation).

CRISPR-Cas 9 system for gene knockout (KO). Single guide RNAs (sgRNAs) targeting human EHZ2 or control (AAVS1) were cloned into lentiCRISPRv2 (Addgene) as previously described (25). HEK293T (ATCC; cat. no. CRL-3216) cells were cultured in DMEM (Thermo Fisher Scientific, Inc.) supplemented with $10 \% \mathrm{FBS}$ and $100 \mathrm{U} / \mathrm{ml}$ penicillin-streptomycin at $37^{\circ} \mathrm{C}$ in an atmosphere containing $5 \% \mathrm{CO}_{2}$. Cells were transfected with $6.7 \mathrm{mg}$ psPAX2 (Addgene, Inc.), $4.1 \mathrm{mg}$ VSV-G (Addgene, Inc.) and $10 \mathrm{mg}$ lentiviral vectors using $60 \mathrm{mg}$ of linear polyethylenimine (Polysciences, Inc.). Subsequently, supernatants containing lentivirus were harvested at $48 \mathrm{~h}$ post-transfection and concentrated by ultracentrifugation $\left(26,000 \mathrm{x}\right.$ g for $2 \mathrm{~h}$ at $\left.4^{\circ} \mathrm{C}\right)$. THP-1 cells were plated into 12 -well plates $\left(0.5-3 \times 10^{6}\right.$ cells per well) with medium supplemented with $8 \mathrm{mg} / \mathrm{ml}$ polybrene (Sigma-Aldrich; Merck KGaA) and spin-infected at $700 \mathrm{xg}$ for $2 \mathrm{~h}$ at $37^{\circ} \mathrm{C}(26)$. Cells were used for subsequent experiments 2 days after the transfection procedure. CRISPR sgRNA sequences were as follows: Control sgRNA (AAVS1), 5'-CACCGCTCCCTG GCCACTTTGCAC-3'; EZH2 KO sgRNA\#1, 5'-CACCGTGTT GGGGGTACATTCAGG-3'; EZH2 KO sgRNA\#2, 5'-CAC CGTATCAGAAGGAAATTTCCG-3'; and EZH2 KO sgRNA\#3 5'-CACCGTATGATGGGAAAGTACACG-3'.

Cholesterol efflux assay. THP-1 or HUVEC cells were cultured at $60 \%$ confluence and then incubated with $0.2 \mathrm{mCi} / \mathrm{ml}[3 \mathrm{H}]$ cholesterol (PerkinElmer, Inc.). After incubation for $24 \mathrm{~h}$, cells were washed with cold PBS twice and equilibrated with fresh medium. Equilibrated [3H] cholesterol-labeled cells were then washed with cold PBS and incubated in efflux medium containing RPMI-1640 medium, 0.1\% BSA (EMD Millipore; cat. no. A7906) and human plasma apoA-I $(25 \mu \mathrm{g} / \mathrm{ml})$ for $6 \mathrm{~h}$. The medium was collected and centrifuged at $14,000 \mathrm{x}$ g for at least $10 \mathrm{~min}$ at $4^{\circ} \mathrm{C}$. Total cell-associated radioactivity was determined by dissolving the cells in isopropanol $(96.7 \%$, $200 \mu \mathrm{l})$. Medium and cell-associated [3H] cholesterol was then measured by liquid scintillation counting (22). The $\%$ efflux was calculated using the following equation: [total media count/(total cellular count + total medium count)] x100\% $(22,27)$.

Oil Red O stain. THP-1 cells were cultured in RPMI-1640 medium and treated with PMA $(100 \mathrm{nmol} / \mathrm{l})$ for $48 \mathrm{~h}$. Subsequently, THP-1 cells were washed with PBS and cultured in a serum free medium containing Ox-LDL (50 $\mu \mathrm{g} / \mathrm{ml})$ and human apoA-I $(25 \mu \mathrm{g} / \mathrm{ml}$; Biodesign International, Inc.) for a further $48 \mathrm{~h}$. After fully differentiating into foam cells, the cells were verified by fixing with $4 \%$ paraformaldehyde at room temperature for $30 \mathrm{~min}$, and subsequently staining with $0.5 \%$ Oil Red O and hematoxylin separately at room temperature for $10 \mathrm{~min}$. Hematoxylin was used for counterstaining cell nuclei and cells were photographed at x200 magnification under a light microscope (Olympus DP80 Microscope). Cells stained with Oil Red O were quantified using ImageJ/FIJI platform (National Institutes of Health).

In vivo protocol. Male ApoE ${ }^{-/}$mice $(\mathrm{n}=18$; age, 6-8 weeks; weight $\sim 20 \mathrm{~g}$ ) were purchased from Beijing HFK Bioscience Co., Ltd. and maintained under a specific pathogen-free environment in microisolator cages. Animals were kept at $18-23^{\circ} \mathrm{C}$ at $40-60 \%$ humidity under a $12 \mathrm{~h} \mathrm{light/dark} \mathrm{cycle.} \mathrm{The} \mathrm{mice}$ were separated into the control group (chow diet + DMSO/PBS injection), vehicle group (high-fat diet + DMSO/PBS injection) and treatment group (high-fat diet + GSK126 treatment), with 
six mice in each group. The mice in the control group were fed a chow diet, whereas the mice in the vehicle and treatment groups were fed a high-fat diet ( $21 \%$ fat, $0.15 \%$ cholesterol; Mediscience Diets Co., Ltd.). All animals had free access to foods. In addition, the mice in the treatment group received a daily intraperitoneal injection of GSK126 at a dose of $50 \mathrm{mg} / \mathrm{kg} /$ day (mouse body weight) for 10 weeks. The mice in the vehicle group were administered a daily intraperitoneal injection of $20 \% \mathrm{DMSO} / \mathrm{PBS}$ (volume, $0.1 \mathrm{ml}$ ). Animals were sacrificed at 16-18 weeks of age. $\mathrm{CO}_{2}$ was supplied in a precisely regulated and purified form. Flow meter was used in order to maintain a gradual fill/displacement rate of $10-30 \%$ of chamber volume per min. $\mathrm{CO}_{2}$ flow was maintained for at least $1 \mathrm{~min}$ after respiratory arrest. Following sacrifice by inhalation of $\mathrm{CO}_{2}$, the animals' heart tissue samples were collected, embedded in the OCT gel without fixation and stored at $-80^{\circ} \mathrm{C}$ immediately. All animal procedures were approved by the Animal Ethics Committee of Dalian Medical University (Dalian, China). All in vivo experiments were performed in accordance with the national legislation and institutional guidelines (approval no. 17-007).

Atherosclerotic lesion analysis. Frozen sections (OCT gel frozen samples; $8 \mu \mathrm{m}$ sections) of the aortic root were stained with Oil Red $\mathrm{O}$ and $\mathrm{H} \& \mathrm{E}$ as previously described. The plaque area in the aortic root sections was then measured using computer-assisted image quantification with Image Pro Plus 6.0 (Media Cybernetics, Inc.). Images were captured using an Olympus fluorescent microscope (DP80; Olympus Corp). Frozen sections were also stained with Masson's trichrome at room temperature for $30 \mathrm{~min}$ according to manufacturer's instructions to measure the collagen content in plaques. All staining solutions were obtained from BASO Precision Optics Ltd.

Immunohistochemistry. Frozen sections of the aortic root $(8 \mu \mathrm{m})$ were first fixed at room temperature for $20 \mathrm{~min}$ in methanol (98\%, Sigma-Aldrich; Merck KGaA), then were incubated with $3 \% \mathrm{H}_{2} \mathrm{O}_{2}$ for $15 \mathrm{~min}$ at room temperature (OriGene Technologies, Inc.), air-dried for $15 \mathrm{~min}$ and incubated with $10 \%$ goat serum (OriGene Technologies, Inc.) for $~ 30 \mathrm{~min}$ at room temperature. The frozen sections were incubated with anti-LOX-1 (1:200 dilution) and anti-VCAM1 (1:400 dilution) antibodies overnight at $4^{\circ} \mathrm{C}$ overnight. Images were captured using an Olympus fluorescent microscope (DP80; Olympus Corp). Five immunohistochemistry sections were quantified using the ImageJ/FIJI platform (National Institutes of Health).

Lipoprotein uptake assay. THP-1 cells were cultured in RPMI-1640 medium and treated with PMA for $48 \mathrm{~h}$. Subsequently, the cells were treated with GSK126 $(5 \mu \mathrm{M})$ for $36 \mathrm{~h}$ at $37^{\circ} \mathrm{C}$ in a $\mathrm{CO}_{2}$ incubator. THP-1 macrophages were then washed with PBS and cultured in serum-free RPMI-1640 medium. Following overnight fasting, the macrophages were washed with PBS again and cultured in medium with or without Ox-LDL (100 $\mu \mathrm{g} / \mathrm{ml}$; Guangzhou Yiyuan Biotechnology Co., Ltd.) for $48 \mathrm{~h}$. After incubation, macrophages were fixed in methanol at room temperature (98\%, Sigma-Aldrich; Merck $\mathrm{KGaA}$ ) for $30 \mathrm{~min}$ and stained with Oil Red $\mathrm{O}$ for $20 \mathrm{~min}$ at room temperature and observed under a DP80 fluorescent microscope at x200 magnification. Experiments were repeated in triplicate in each group.

Western blot analysis. Cell lysates of THP-1 cells were isolated using a Total Protein Extraction kit from Nanjing KeyGen Biotech. Co., Ltd., following the manual provided by the manufacturing company. The protein concentration was determined using a PierceTM BCA Protein Assay kit (Thermo Fisher Scientific, Inc.) following the manufacturer's protocols. Denatured proteins $(30-50 \mu \mathrm{g})$ were separated by $12 \%$ SDS-polyacrylamide gel electrophoresis for $120 \mathrm{~min}$ at $120 \mathrm{~V}$ in electrophoretic buffer solution. The proteins were then transferred to PVDF membranes (EMD Millipore) for $90 \mathrm{~min}$ at $400 \mathrm{~mA}$. Following blocking with $5 \%$ milk/Tris-buffered saline (w/v; Sigma-Aldrich; Merck KGaA) at $25^{\circ} \mathrm{C}$ for $1 \mathrm{~h}$, the membranes were incubated with primary antibodies at $4^{\circ} \mathrm{C}$ overnight, including anti-LOX-1 (dilution, 1:1,000), anti- $\beta$-actin (dilution, 1:1,000), anti-histone H3 (dilution, 1:500), anti-H3K27me3 (dilution, 1:1,000), anti-ABCA1 (dilution, 1:1,000) and anti-EZH2 (dilution, 1:1,000). The membranes were then washed with TBST (1\% Tween-20) and incubated with horseradish peroxidase-conjugated goat anti-rabbit IgG (cat. no. sc-2004; dilution, 1:10,000) or horseradish peroxidase-conjugated goat anti-mouse IgM (cat. no. sc-2064; dilution, 10,000) secondary antibodies obtained from Santa Cruz Biotechnology, Inc., and Texas Red-X conjugated goat anti-rat IgG secondary antibody (dilution, 1:200; Thermo Fisher Scientific, Inc.; cat. no. T-6392) for $1 \mathrm{~h}$ at room temperature. The blots were then treated with the WesternBright ECL kit (Advansta Inc.) and visualized using the Bio-Rad imaging system (Bio-Rad Laboratories, Inc.) and analyzed using Image Pro Plus 6.0 (Media Cybernetics, Inc.).

Statistical analysis. Statistical analysis was performed using SPSS version 19.0 statistical software (IBM Corp.) and GraphPad Prism 7 (GraphPad Software). All data are presented as the mean $\pm \mathrm{SD}$. A one-way ANOVA was performed to analyze multiple group comparisons of quantitative data. Bonferroni post hoc-tests were used after one-way ANOVAs. $\mathrm{P}<0.05$ was considered to indicate a statistically significant difference.

\section{Results}

EZH2 inhibition markedly attenuated lipoprotein uptake and reduced foam cell formation in human THP-1 monocytes. To gain molecular insights into the therapeutic effect of EZH2 inhibition on the development of atherosclerosis, the role of EZH2 was examined using an in vitro model of atherosclerosis, specifically using THP-1 human monocytes, which are commonly used for in vitro studies into atherosclerosis (10). The present study performed KO of the EZH2 gene in THP-1 monocytes and it was observed that EZH2 KO significantly decreased the abundance of H3K $27 \mathrm{me} 3$ in THP-1 cells compared to control cells (Fig. 1A). Meanwhile, administration of GSK126, a specific EZH2 inhibitor, completely abolished H3K27 methylation in THP-1 cells but did not affect the protein expression levels of EZH2 (Fig. 1A).

The uptake of Ox-LDL by macrophages and subsequent formation of foam cells is initiated by the development of 
A
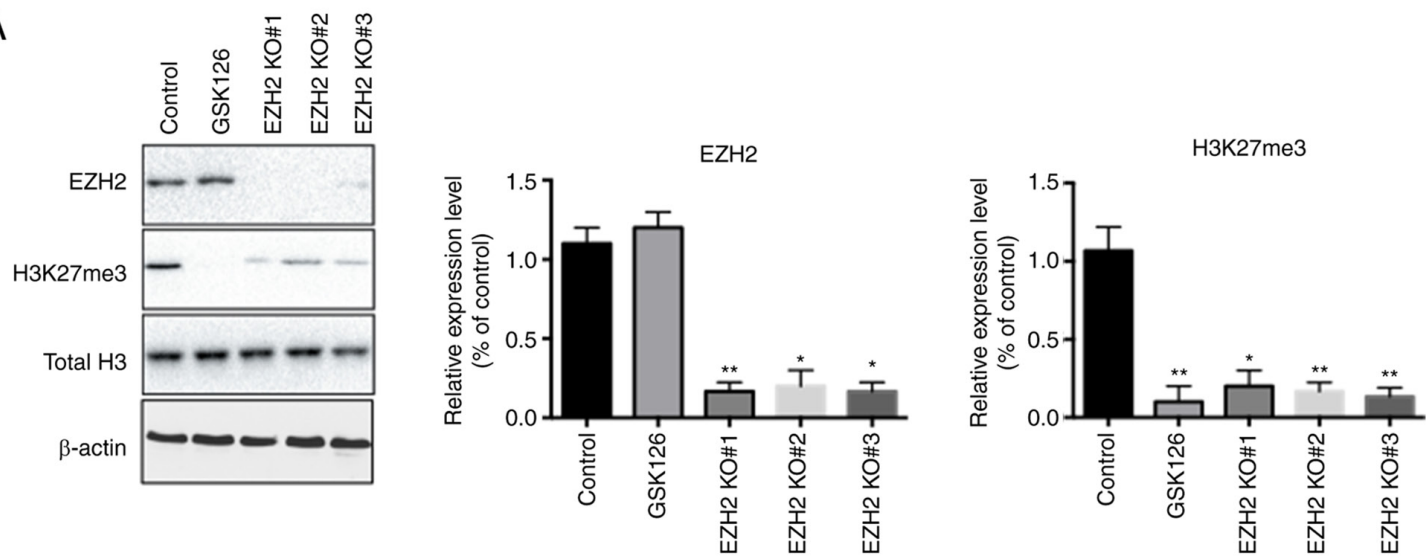

B

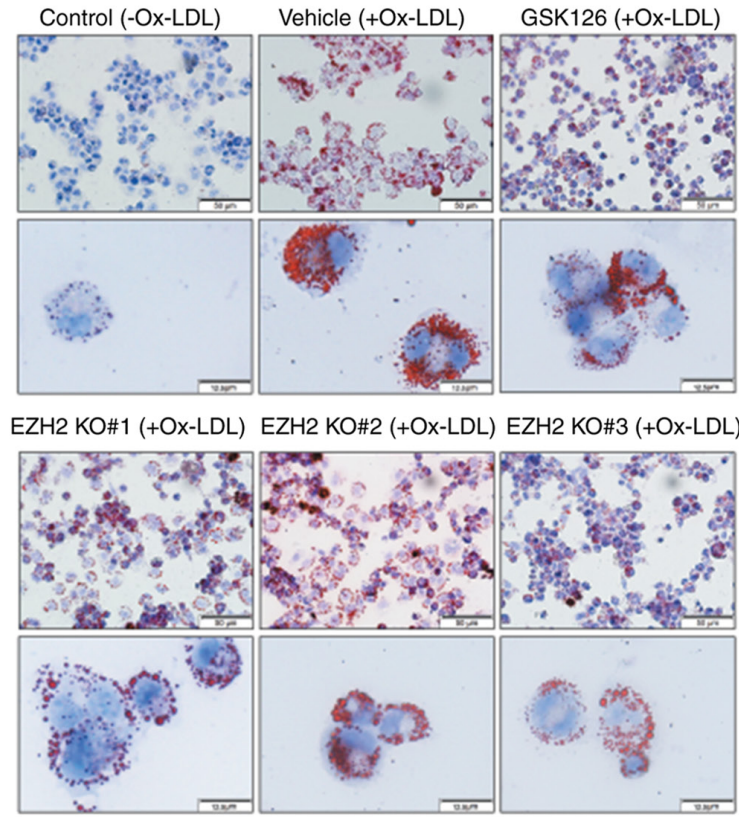

C

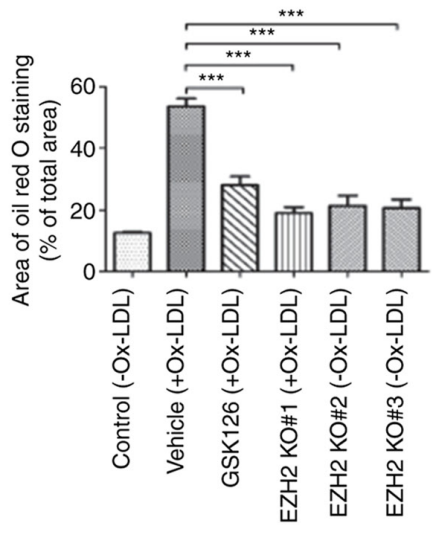

D

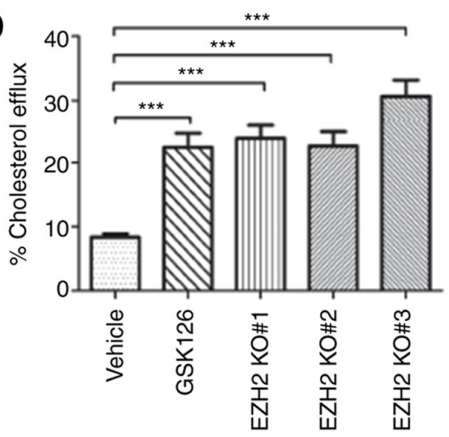

$\mathrm{E}$
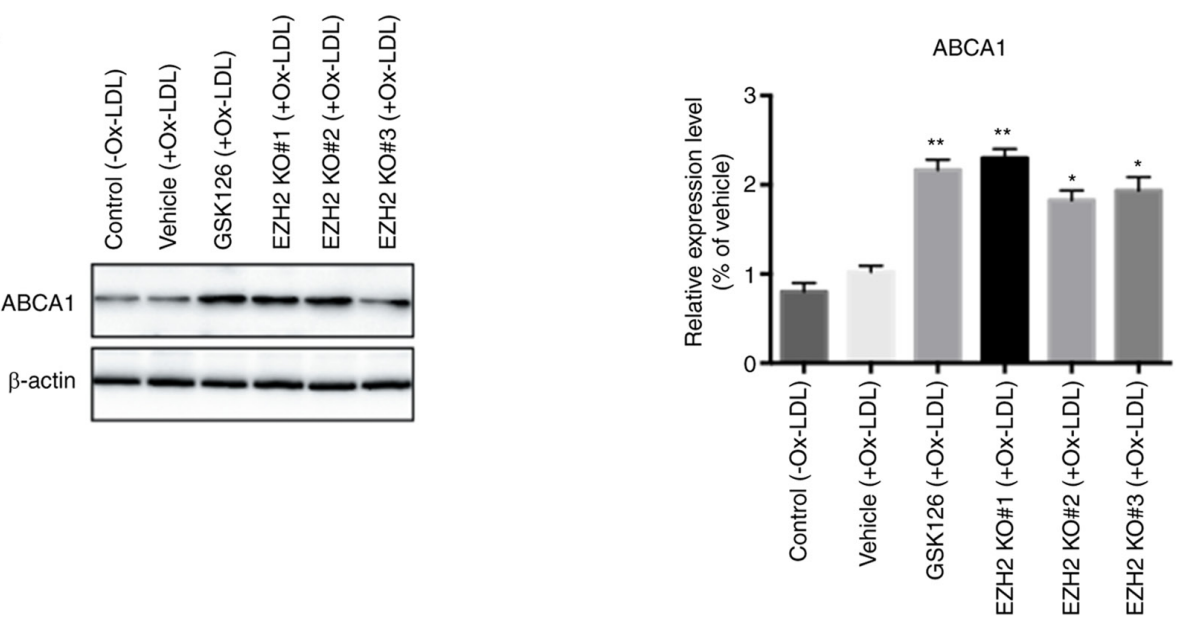

Figure 1. EZH2 inhibition reduces macrophage foam cell formation. (A) Western blotting of EZH2 expression in THP-1 cells transduced with single gRNA targeting EZH2. EZH2 expression levels and H3K27me3 levels of THP-1 cells that were transfected with a control vector or sgRNAs targeting EZH2; or treated with GSK126 $(5 \mu \mathrm{M})$, as detected using western blotting. (B) THP-1 cells were stained for lipids using Oil Red O and nuclei were counterstained using hematoxylin. (C) Quantification of the area stained with Oil Red O showed decreased uptake of Ox-LDL in EZH2-null and GSK126-treated THP-1 cells $(5 \mu \mathrm{M})$ compared with vehicle-treated THP-1 cells. (D) Effect of EZH2 inhibition on cholesterol efflux in THP-1 cells treated with Ox-LDL. (E) Western blotting of ABCA1 expression levels in THP-1 cells. Results are presented as the mean $\pm \mathrm{SD}(\mathrm{n}=3)$. ${ }^{*} \mathrm{P}<0.05,{ }^{* *} \mathrm{P}<0.01$ and ${ }^{* * * *} \mathrm{P}<0.001$. ABCA1, ATP-binding cassette transporter A1; EZH2, enhancer of zeste homolog 2; H3K27me3, trimethylation of histone H3 at Lys 27; KO, knockout; Ox-LDL, oxidized low-density lipoprotein; sgRNA. 


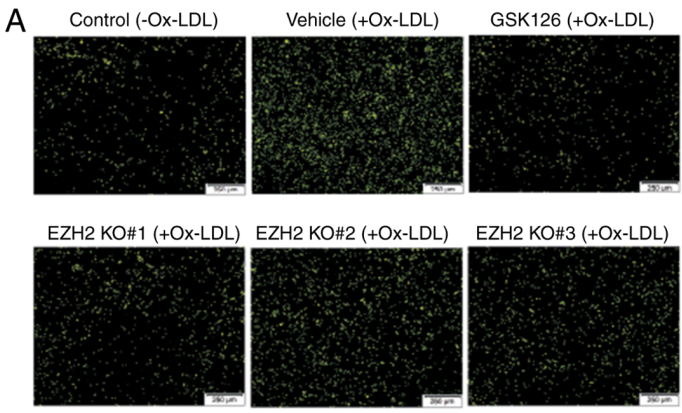

C

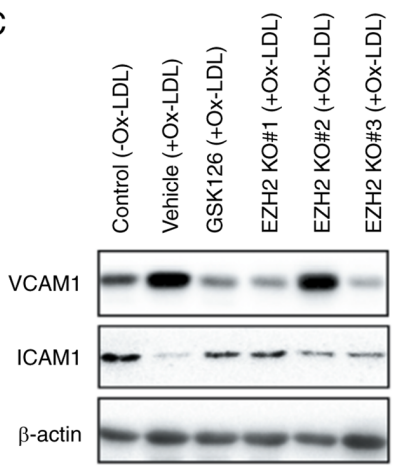

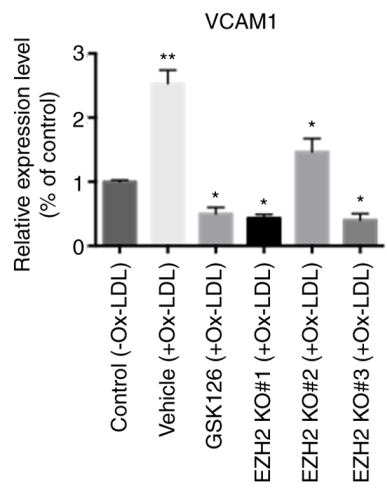

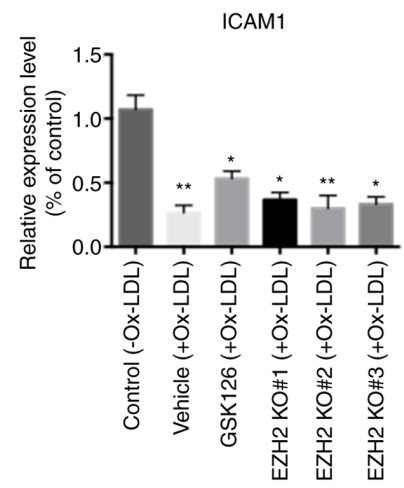

Figure 2. EZH2 inhibition blocks Ox-LDL induced monocyte adhesion of HUVECs. (A) Decreased THP-1 monocyte adhesion to HUVECs in EZH2-null and GSK126-treated THP-1 cells ( $5 \mu \mathrm{M})$ compared with vehicle-treated THP-1 cells. THP-1 cells were labeled with carboxyfluorescein. (B) Quantification of adherent THP-1 cells (green) on HUVECs. (C) Western blotting of VCAM1 and ICAM1 expression levels in EZH2-null and GSK126-treated THP-1 cells post Ox-LDL stimulation. Results are presented as the mean \pm SD $(n=3)$. ${ }^{*} \mathrm{P}<0.05$ and ${ }^{* *} \mathrm{P}<0.01$. EZH2, enhancer of zeste homolog 2; ICAM1, intercellular adhesion molecule 1; KO, knockout; Ox-LDL, oxidized low-density lipoprotein; VCAM1, vascular cell adhesion molecule 1.

atherosclerosis (28). To evaluate whether EZH2 regulates lipid uptake and foam cell formation, THP-1 cells were incubated with Ox-LDL or vehicle for $48 \mathrm{~h}$ after $\mathrm{KO}$ or pharmacological inhibition of EZH2. Compared with the vehicle treatment, the accumulation of cytoplasmic lipid droplets, as determined by Oil Red O staining, was visibly reduced in the GSK126 treated and EZH2-null THP-1 cells, implying that foam cell formation is dependent of EZH2 (Fig. 1B and C). Furthermore, the present study determined whether the inhibition of EZH2 with sgRNA or treatment with GSK126 altered the cholesterol efflux in THP-1 macrophage-derived foam cells. Both GSK126 treatment and EZH2-gRNA lentiviral infection significantly increased cellular cholesterol efflux (Fig. 1D). To further understand the inhibitory effect of EZH2 inhibition on foam cell formation, the protein expression levels of ATP Binding Cassette Subfamily A Member 1 (ABCA1) were examined, a major regulator of cellular cholesterol and phospholipid homeostasis in human beings (22). It was found that the protein expression levels of ABCA1 were significantly increased following EZH2 inhibition by $\mathrm{EZH} 2 \mathrm{KO}$ and GSK126 in THP-1 cells as evidenced by western blotting (Fig. 1E). mRNA expression levels of other important regulators of cellular cholesterol homeostasis were also measured, including CD36 and ATP Binding Cassette Subfamily G Member 1 (ABCG1) in THP-1 cells treated by GSK126 or vehicle. No significant differences were observed to the expression levels of CD36 and ABCG1 (data not shown). Taken together, these data suggested that an EZH2-dependent epigenetic regulation is involved in foam cell formation, likely through the upregulation of ABCA1 at the protein level in THP-1 cells.

Inhibition of EZH2 significantly blocks Ox-LDL induced monocyte adhesion. Monocyte adhesion to the vascular wall is another crucial step in the development of atherosclerosis (29). The present study assessed whether EZH2 KO or GSK126 could decrease the extent of monocyte adhesion to HUVECs. THP-1 and HUVECs were co-cultured, then the number of BCECF-AM-labeled THP-1 monocytes bound to HUVECs following Ox-LDL stimulation was counted. Following treatment with GSK126 and EZH2 KO, the number of THP-1 monocytes adhered to HUVECs was significantly reduced when compared to those in vehicle-treated wells (Fig. 2A and B). Furthermore western blotting analysis revealed that inhibition of EZH2 activity significantly downregulated the protein expression levels of VCAM1 and upregulated ICAM1 levels (Fig. 2C), both of which have been previously implicated to affect the recruitment of monocytes to endothelial cells (30). However, VCAM1 is unique in that its expression is largely restricted to lesions and lesion-predisposed regions, whereas ICAM1 expression extends into uninvolved healthy aorta and 

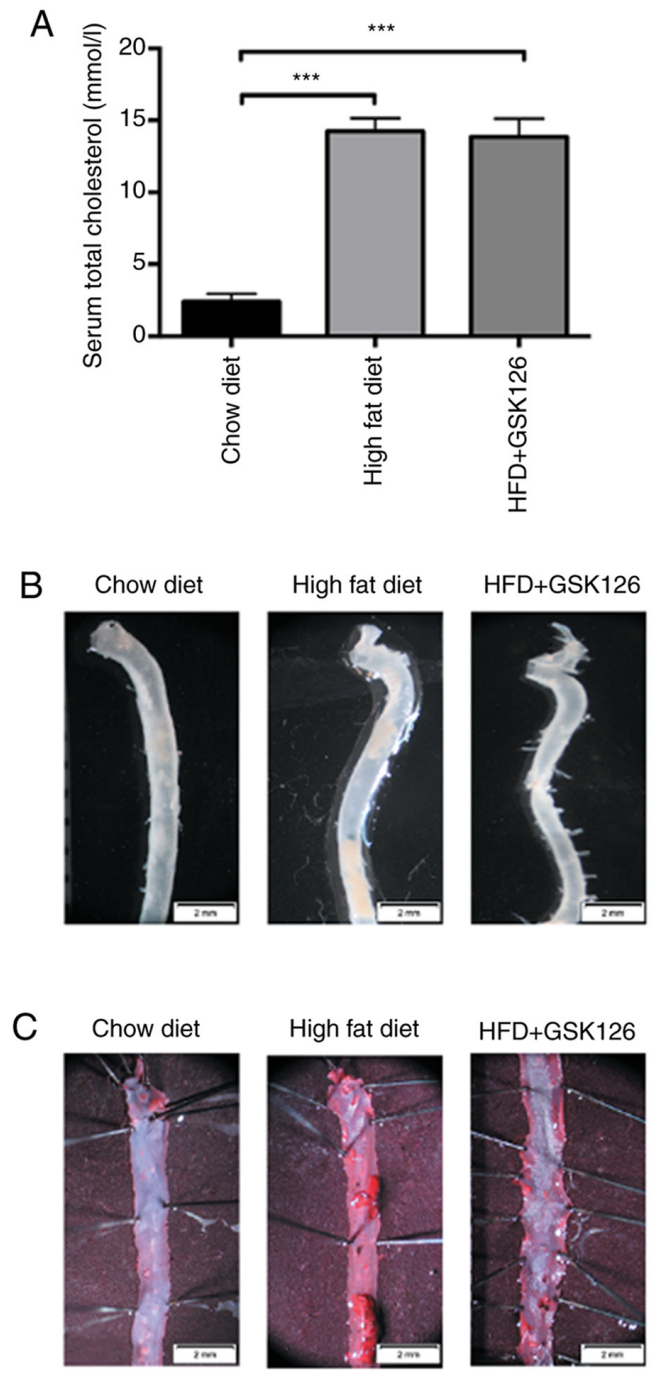

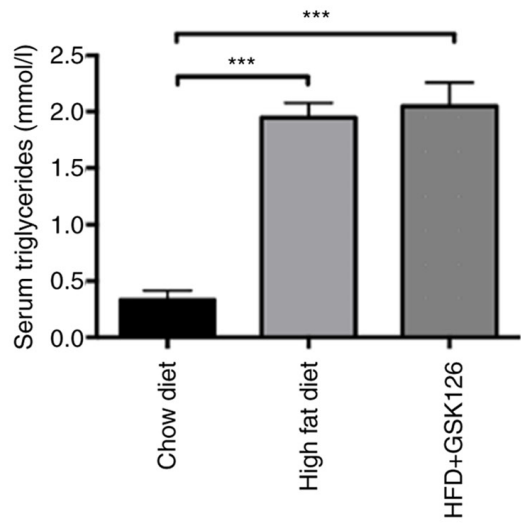

E

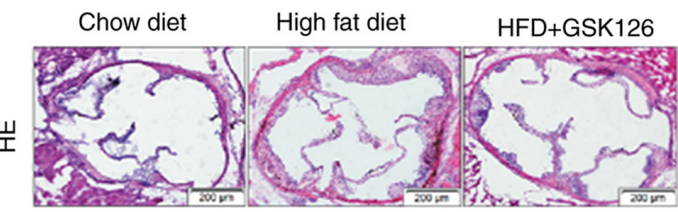

$\mathrm{F}$

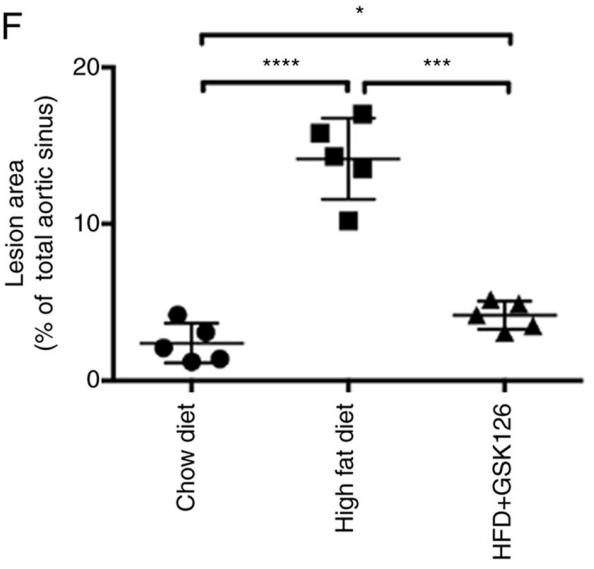

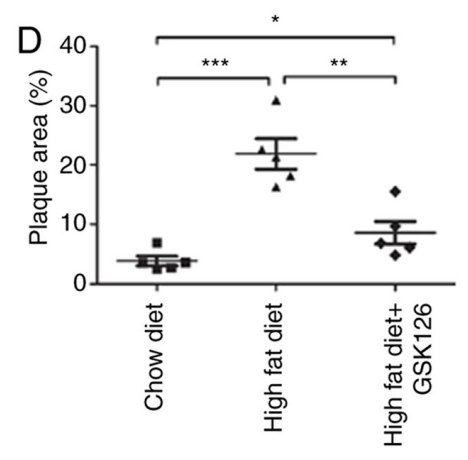

Figure 3. GSK126 attenuates the development of atherosclerotic lesions in $\mathrm{ApoE}^{-/}$mice. (A) Serum total cholesterol and triglyceride levels were measured. (B-C) En face Oil Red O staining of the thoracic aorta and (D) quantification of plaque area (n=5). Magnification, x4. (E) Representative photomicrographs of Oil Red O staining from mice with comparable lesion sizes (F) Quantification of lesion sizes from the various treatment groups ( $\mathrm{n}=5$ ). Results are presented as the mean $\pm \mathrm{SD}$. Magnification, $\mathrm{x} 10 .{ }^{*} \mathrm{P}<0.05,{ }^{* *} \mathrm{P}<0.01,{ }^{* * *} \mathrm{P}<0.001$. and ${ }^{* * * * *} \mathrm{P}<0.001 \mathrm{ApoE}$, apolipoprotein $\mathrm{E} ; \mathrm{HFD}$, High fat diet; HE, hematoxylin and eosin.

lesion-protected regions (31). The difference in expression patterns suggest different functions for VCAM1 and ICAM1 in lesion initiation. In addition, the expression of VCAM1 was significantly reduced in THP-1 cells treated with GSK126, providing a molecular basis for the effects of EZH2 on Ox-LDL stimulated monocyte adhesion. Taken together, these findings provide direct evidence that methylation of $\mathrm{H} 3 \mathrm{~K} 27$ contributes to monocyte adhesion in an EZH2-dependent manner.
GSK126 attenuates the development of atherosclerosis in ApoE ${ }^{-1-}$ mouse models fed a high-fat diet. To address the role of EZH2 inhibition on the development of atherosclerosis, male $A p o E^{-/-}$mice, which were fed an atherogenic high-fat diet, were treated with GSK126 $(50 \mathrm{mg} / \mathrm{kg} / \mathrm{day})$ or vehicle $(20 \%$ DMSO/PBS, $0.1 \mathrm{ml})$ via a daily intraperitoneal injection for a period of 10 weeks. Peripheral blood and aortas from animals were collected for cholesterol testing and Oil 
A
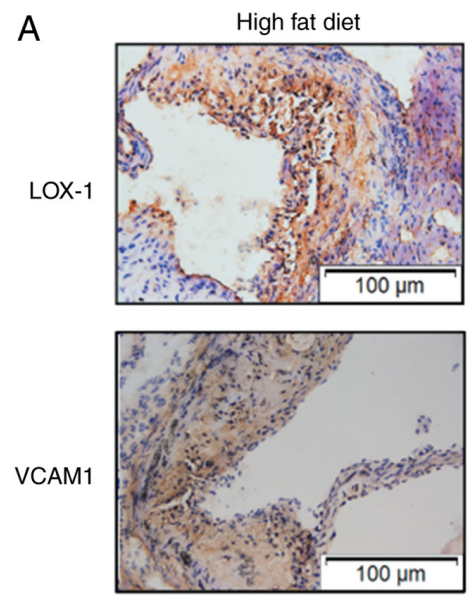

HFD+GSK126
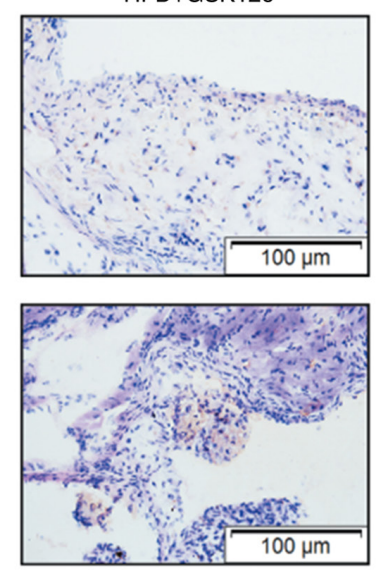

$\mathrm{B}$

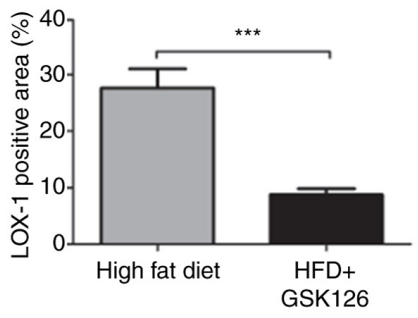

C

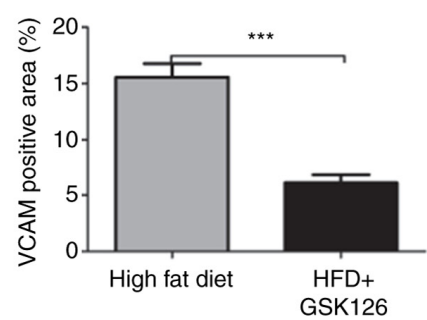

Figure 4. GSK126 decreases atherosclerosis development by downregulating LOX-1 and VCAM1 expression levels in plaques. (A) Representative photomicrographs of LOX-1 and VCAM1 expression in lesions following anti-LOX-1 and anti-VCAM1 antibody staining. Magnification, x40. (B) Quantitative analysis of LOX-1 expression area in the lesions $(n=5)$. (C) Quantitative analysis of VCAM1 expression area in the lesions ( $=5)$. Results are presented as the mean \pm SD. ${ }^{* * *} \mathrm{P} \leq 0.001$. HFD, High fat diet; VCAM1, vascular cell adhesion molecule 1; LOX-1, oxidized low-density lipoprotein receptor 1 .

Red O staining, respectively. Consistent with the aforementioned in vitro findings, $A p o E^{-/-}$mice fed with a high-fat diet exhibited a significant increase in cholesterol and triglyceride levels, but a a significant decrease with GSK126 treatment, as compared with the animals fed a chow diet (Fig. 3A). Subsequently, whole mouse aortas were harvested (Fig. 3B). En face Oil Red $\mathrm{O}$ staining was performed on the thoracic aortas isolated from $A p o E^{-/-}$mice fed an atherogenic high-fat diet and treated with GSK126 or vehicle Mice treated with GSK126 exhibited significantly reduced atherosclerotic plaque size (Fig. 3C and D), indicating that GSK126 exerts a protective effect, suppressing atherosclerotic plaque development in mouse models. H\&E staining of serial cross sections of aortic roots also revealed reduced atherosclerotic lesion sizes in the GSK126-treated animals, as compared with the vehicle-treated mice (Fig. 3E and F). These results indicated that GSK126 has the ability to attenuate the progression of atherosclerosis in atherosclerosis-prone models.

GSK126 reduces macrophage recruitment to atherosclerotic plaques. Previous studies have demonstrated that overexpression of lectin-type oxidized LOX-1, a macrophage and endothelial cell receptor for Ox-LDL $(32,33)$, in animal models of atherosclerosis, enhances the levels of valvular accumulation of Ox-LDL, thereby favouring endothelial dysfunction, vascular inflammation and plaque formation (34). According to our previous studies (10), chow fat diet does not induce atherosclerosis and Lox-1 expression in $A p o E^{-/-}$mice. In order to investigate whether GSK126 modulates the expression levels of Lox-1 during plaque formation in mice, the protein expression levels of Lox-1 in plaques were examined from mice fed a high-fat diet. Immunohistochemical staining of cross sections of the mouse aorta revealed a decreased protein expression of Lox-1 in GSK126-treated animals, as compared with the vehicle-treated control mice (Fig. 4A and B). Upon initiation of plaque formation, Ox-LDL stimulates inflammatory pathways in monocytes and endothelial cells, inducing monocyte adhesion to endothelial cells (35). In line with this concept, the present study observed an increase in VCAM1 expression in GSK126-treated mice compared with mice treated with vehicle, as shown by immunohistochemical staining of aortas (Fig. 4A). The relative VCAM1-positive area in the GSK126-treated mice was significantly decreased compared with that of the vehicle-treated animals (Fig. 4C). These results suggested that GSK126 is sufficient to suppress atherosclerotic plaque formation in mouse models, possibly through the modulation of LOX-1 induced lipid uptake and VCAM1 induced monocyte adhesion to endothelial cells.

\section{Discussion}

Histone modification controls gene expression by changing DNA accessibility or chromatin structure, and plays important roles in a wide range of pathological processes, such as tumorigenesis, atherosclerosis and inflammation (36-38). Recent studies have highlighted the importance of EZH2 histone methyltransferase on the development and progression of atherosclerosis $(21,22,34,37)$. Interestingly, contradictory results have been observed in studies from different groups. For example, Greißel et al (21) observed significantly increased H3K27 methylation levels in endothelial cells from human early and advanced atherosclerotic plaques. However, other studies have reported a global decrease in H3K27 methylation levels during progression of atherosclerosis $(21,36,39)$. Meanwhile, EZH2 has also been reported to recruit DNA methyltransferase 1 to the promoter region of the ABCA1 gene, which in turn increases methylation of $\mathrm{CpG}$ dinucleotides at the ABCA1 promoter and consequently suppresses its transcriptional activity (22).

Additionally, EZH2 has been shown to play a crucial role in macrophage/microglial activation, mediating the expression of toll-like receptor-induced proinflammatory genes, such as IL-6, IL-12b, TNF- $\alpha$, CeC motif chemokine ligand 2 and $\mathrm{C}-\mathrm{X}-\mathrm{C}$ motif chemokine 10. EZH2 ChIP-seq analysis 
has identified that the expression of suppressor of cytokine signaling 3, an anti-inflammatory gene, is regulated by EZH2 mediated H3K27me3. Furthermore, EZH2 deficiency significantly attenuates the expression of proinflammatory genes at both the mRNA and protein levels, and therefore diminishes macrophage/microglial activation and attenuates the autoimmune inflammation (40). These results suggest that EZH2 may be a potential candidate for anti-atherosclerosis therapy.

Development of EZH2 small molecule inhibitors has been an active area of investigation (41). Pharmaceutical companies have been increasingly developing compounds and promising preclinical results have been obtained for the treatment of breast cancer, with early results suggesting a potential treatment utility (42). GSK126 is a potent and specific methyltransferase inhibitor which is highly selective for the histone methyltransferase EZH2, with a very low $\mathrm{IC}_{50}$ of $9.9 \mathrm{nM}$ (40). GSK126 has been shown to have a 1,000-fold selectivity for EZH2 over other human methyltransferases $(43,44)$. However, it has not yet been clarified whether inhibition of EZH2 by a small-molecule inhibitor has anti-atherosclerotic effects. The present study provided evidence that EZH2 plays a critical role in the initiation of atherosclerosis, specifically in foam cell formation and monocyte adhesion. Pharmacological inhibition of EZH2 appears to be antiatherogenic in ApoE-/- mice fed a high-fat diet. Additionally, pharmacological inhibition of EZH2 by GSK126, as shown in the present study, or by GSK343 as previously shown (42), significantly decreases the expression levels of proatherogenic genes, including VCAM1 and LOX-1 in macrophages or atherosclerotic plaques, thereby attenuating the ability of lipid uptake and monocyte adhesion to arterial walls (45). These results suggested that GSK126 is a potential drug candidate for the treatment of atherosclerosis.

In conclusion, the present study identified that EZH2, as a key epigenetic regulator, promotes the development of atherosclerotic lesions and further demonstrated that the EZH2 inhibitor GSK126 is able to effectively reduce atherosclerosis in $A p o E^{-/-}$mice by suppressing foam cell formation and monocyte adhesion. The present study also provided novel insights into the protective effects of EZH2 inhibition on blocking lipid uptake by upregulating the expression of ABCA1 and reducing monocyte adhesion by downregulating the expression of VCAM1. These results suggested that EZH2 may be considered a potential therapeutic target for the prevention of atherosclerotic disease progression.

\section{Acknowledgements}

Not applicable.

\section{Funding}

This study was supported by funds from the Affiliated Zhongshan Hospital of Dalian University and the National Natural Science Foundation of China (grant no. 81372858).

\section{Availability of data and materials}

The datasets used and/or analyzed during the present study are available from the corresponding author on reasonable request.

\section{Authors' contributions}

$\mathrm{XWa}, \mathrm{XWe}$, and LX contributed in the conception and design of the study. XWe, YZ and KW contributed in data acquisition, analysis and interpretation of the data, statistical analysis, manuscript drafting, and critical revision of the manuscript for important intellectual content. XWa and XWe are responsible for the authenticity of all data. All authors read and approved the final manuscript.

\section{Ethics approval and consent to participate}

Animal procedures were approved (approval no. 17-007) by the Animal Ethics Committee of the Affiliated Zhongshan Hospital of Dalian University (Dalian, China). All in vivo experiments were performed in accordance with national legislation and institutional guidelines.

\section{Patient consent for publication}

Not applicable.

\section{Competing interests}

The authors declare that they have no competing interests.

\section{References}

1. Libby P, Ridker PM and Hansson GK: Progress and challenges in translating the biology of atherosclerosis. Nature 473: 317-325, 2011.

2. Libby P: Inflammation in atherosclerosis. Nature 420: 868-874, 2002.

3. Hansson GK and Libby P: The immune response in atherosclerosis: A double-edged sword. Nat Rev Immunol 6: 508-519, 2006.

4. Hansson GK: Inflammation, atherosclerosis, and coronary artery disease. N Engl J Med 352: 1685-1695, 2005.

5. Jonasson L, Holm J, Skalli O, Bondjers G and Hansson GK: Regional accumulations of $\mathrm{T}$ cells, macrophages, and smooth muscle cells in the human atherosclerotic plaque. Arteriosclerosis 6: 131-138, 1986.

6. Stary HC, Chandler AB, Dinsmore RE, Fuster V, Glagov S, Insull W Jr, Rosenfeld ME, Schwartz CJ, Wagner WD and Wissler RW: A definition of advanced types of atherosclerotic lesions and a histological classification of atherosclerosis. A report from the Committee on Vascular Lesions of the Council on Arteriosclerosis, American Heart Association. Arterioscler Thromb Vasc Biol 15: 1512-1531, 1995.

7. Ross R: Atherosclerosis is an inflammatory disease. Am Heart J 138: S419-S420, 1999.

8. Kolodgie FD, Gold HK, Burke AP, Fowler DR, Kruth HS, Weber DK, Farb A, Guerrero LJ, Hayase M, Kutys R, et al: Intraplaque hemorrhage and progression of coronary atheroma. N Engl J Med 349: 2316-2325, 2003.

9. van der Wal AC, Das PK, Bentz van de Berg D, van der Loos CM and Becker AE: Atherosclerotic lesions in humans. In situ immunophenotypic analysis suggesting an immune mediated response. Lab Invest 61: 166-170, 1989.

10. Wang XQ, Wan HQ, Wei XJ, Zhang Y and Qu P: CLI-095 decreases atherosclerosis by modulating foam cell formation in apolipoprotein E-deficient mice. Mol Med Rep 14: 49-56, 2016.

11. Opar A: Where now for new drugs for atherosclerosis? Nat Rev Drug Discov 6: 334-335, 2007.

12. Wierda RJ, Geutskens SB, Jukema JW, Quax PHA and van den Elsen PJ: Epigenetics in atherosclerosis and inflammation. J Cell Mol Med 14 (6A): 1225-1240, 2010.

13. Sharma P, Kumar J, Garg G, Kumar A, Patowary A,Karthikeyan G, Ramakrishnan L, Brahmachari V and Sengupta S: Detection of altered global DNA methylation in coronary artery disease patients. DNA Cell Biol 27: 357-365, 2008. 
14. Yi-Deng J, Tao S, Hui-Ping Z, Jian-Tuan X, Jun C, Gui-Zhong L and Shu-Ren W: Folate and ApoE DNA methylation induced by homocysteine in human monocytes. DNA Cell Biol 26: 737-744, 2007.

15. Delaney C, Garg SK, Fernandes C, Hoeltzel M, Allen RH, Stabler S and Yung R: Maternal diet supplemented with methyl-donors protects against atherosclerosis in F1 ApoE(-/-) mice. PLoS One 8: e56253, 2013.

16. Castro R, Rivera I, Struys EA, Jansen EE, Ravasco P, Camilo ME, Blom HJ, Jakobs $\mathrm{C}$ and Tavares de Almeida I: Increased homocysteine and $\mathrm{S}$-adenosylhomocysteine concentrations and DNA hypomethylation in vascular disease. Clin Chem 49: 1292-1296, 2003.

17. Kim M, Long TI, Arakawa K, Wang R, Yu MC and Laird PW: DNA methylation as a biomarker for cardiovascular disease risk. PLoS One 5: e9692, 2010.

18. Kim J, Kim JY, Song KS, Lee YH, Seo JS, Jelinek J, Goldschmidt-Clermont PJ and Issa JP: Epigenetic changes in estrogen receptor beta gene in atherosclerotic cardiovascular tissues and in-vitro vascular senescence. Biochim Biophys Acta 1772: 72-80, 2007.

19. Jiang W, Agrawal DK and Boosani CS: Cell specific histone modifications in atherosclerosis (Review). Mol Med Rep 18: $1215-1224,2018$

20. Khyzha N, Alizada A, Wilson MD and Fish JE: Epigenetics of Atherosclerosis: Emerging Mechanisms and Methods. Trends Mol Med 23: 332-347, 2017.

21. Greißel A, Culmes M, Burgkart R,Zimmermann A, Eckstein $\mathrm{HH}$ Zernecke A and Pelisek J: Histone acetylation and methylation significantly change with severity of atherosclerosis in human carotid plaques. Cardiovasc Pathol 25: 79-86, 2016.

22. Lv YC, Tang YY, Zhang P, Wan W, Yao F, He PP, Xie W, Mo ZC, Shi JF, Wu JF, et al: Histone methyltransferase enhancer of Zeste Homolog 2-mediated ABCA1 promoter DNA methylation contributes to the progression of atherosclerosis. PLoS One 11: e0157265, 2016

23. Xu S, Xu Y, Yin M, Zhang S, Liu P, Koroleva M, Si S, Little PJ Pelisek J and Jin ZG: Flow-dependent epigenetic regulation of IGFBP5 expression by $\mathrm{H} 3 \mathrm{~K} 27 \mathrm{me} 3$ contributes to endothelial anti-inflammatory effects. Theranostics 8: 3007-3021, 2018.

24. Yap TA, Winter JN, Giulino-Roth L, Longley J, Lopez J, Michot JM, Leonard JP, Ribrag V, McCabe MT, Creasy CL, et al Phase I study of the novel enhancer of Zeste Homolog 2 (EZH2) inhibitor GSK2816126 in patients with advanced hematologic and solid tumors. Clin Cancer Res 25: 7331-7339, 2019.

25. Shalem O, Sanjana NE, Hartenian E, Shi X, Scott DA, Mikkelson T, Heckl D, Ebert BL, Root DE, Doench JG, et al: Genome-scale CRISPR-Cas9 knockout screening in human cells. Science 343: 84-87, 2014

26. Yamauchi T, Masuda T, Canver MC, Seiler M, Semba Y, Shboul M, Al-Raqad M, Maeda M, Schoonenberg VAC, Cole MA, et al: Genome-wide CRISPR-Cas9 Screen Identifies Leukemia-Specific Dependence on a Pre-mRNA Metabolic Pathway Regulated by DCPS. Cancer Cell 33: 386-400.e5, 2018.

27. Low H, Hoang A and Sviridov D: Cholesterol efflux assay. J Vis Exp 61: e3810, 2012

28. Zimetti F, Favari E, Cagliero P, Adorni MP, Ronda N, Bonardi R, Gomaraschi M, Calabresi L, Bernini F and Guardamagna O: Cholesterol trafficking-related serum lipoprotein functions in children with cholesteryl ester storage disease. Atherosclerosis 242: 443-449, 2015.

29. Mestas J and Ley K: Monocyte-endothelial cell interactions in the development of atherosclerosis. Trends Cardiovasc Med 18 228-232, 2008

30. Ley K and Huo Y: VCAM-1 is critical in atherosclerosis. J Clin Invest 107: 1209-1210, 2001.
31. Iiyama K, Hajra L, Iiyama M, Li H, DiChiara M, Medoff BD and Cybulsky MI: Patterns of vascular cell adhesion molecule-1 and intercellular adhesion molecule-1 expression in rabbit and mouse atherosclerotic lesions and at sites predisposed to lesion formation. Circ Res 85: 199-207, 1999.

32. Moriwaki H, Kume N, Sawamura T, Aoyama T, Hoshikawa H, Ochi H, Nishi E, Masaki T and Kita T: Ligand specificity of LOX-1, a novel endothelial receptor for oxidized low density lipoprotein. Arterioscler Thromb Vasc Biol 18: 1541-1547, 1998.

33. Sawamura T, Kume N, Aoyama T, Moriwaki H, Hoshikawa H, Aiba Y, Tanaka T, Miwa S, Katsura Y, Kita T, et al: An endothelial receptor for oxidized low-density lipoprotein. Nature 386: 73-77, 1997.

34. Akhmedov A, Rozenberg I, Paneni F, Camici GG, Shi Y, Doerries C, Sledzinska A, Mocharla P, Breitenstein A, Lohmann C, et al: Endothelial overexpression of LOX-1 increases plaque formation and promotes atherosclerosis in vivo. Eur Heart J 35: 2839-2848, 2014.

35. Frostegård J, Nilsson J, Haegerstrand A, Hamsten A, Wigzell H and Gidlund M: Oxidized low density lipoprotein induces differentiation and adhesion of human monocytes and the monocytic cell line U937. Proc Natl Acad Sci USA 87: 904-908, 1990.

36. Greißel A, Culmes M, Napieralski R, Wagner E, Gebhard H, Schmitt M, Zimmermann A, Eckstein HH, Zernecke A and Pelisek J: Alternation of histone and DNA methylation in human atherosclerotic carotid plaques. Thromb Haemost 114: 390-402, 2015.

37. Xu K, Wu ZJ, Groner AC, He HH, Cai C, Lis RT, Wu X, Stack EC, Loda M, Liu T, et al: EZH2 oncogenic activity in castration-resistant prostate cancercells is Polycomb-independent. Science 338: 1465-1469, 2012.

38. Liu Y, Peng J, Sun T, Li N, Zhang L, Ren J, Yuan H, Kan S, Pan Q, Li X, et al: Epithelial EZH2 serves as an epigenetic determinant in experimental colitis by inhibiting TNF $\alpha$-mediated inflammation and apoptosis. Proc Natl Acad Sci USA 114: E3796-E3805, 2017.

39. Wierda RJ, Rietveld IM, van Eggermond MCJA, Belien JAM, van Zwet EW, Lindeman JHN and van den Elsen PJ: Global histone $\mathrm{H} 3$ lysine 27 triple methylation levels are reduced in vessels with advanced atherosclerotic plaques. Life Sci 129: 3-9, 2015.

40. Zhang X, Wang Y, Yuan J, Li N, Pei S, Xu J, Luo X, Mao C, Liu J, Yu T, et al: Macrophage/microglial Ezh2 facilitates autoimmune inflammation through inhibition of Socs3. J Exp Med 215: 1365-1382, 2018

41. Villanueva MT: Anticancer drugs: All roads lead to EZH2 inhibition. Nat Rev Drug Discov 16: 239, 2017.

42. Kim KH and Roberts CWM: Targeting EZH2 in cancer. Nat Med 22: 128-134, 2016.

43. McCabe MT, Ott HM, Ganji G, Korenchuk S, Thompson C, Van Aller GS, Liu Y, Graves AP, Della Pietra A III, Diaz E, et al: EZH2 inhibition as a therapeutic strategy for lymphoma with EZH2-activating mutations. Nature 492: 108-112, 2012

44. Kaniskan HÜ and Jin J: Chemical probes of histone lysine methyltransferases. ACS Chem Biol 10: 40-50, 2015.

45. Zhou J, Huang S, Wang Z, Huang J, Xu L, Tang X, Wan YY, Li QJ, Symonds ALJ, Long H, et al: Targeting EZH2 histone methyltransferase activity alleviates experimental intestinal inflammation. Nat Commun 10: 2427, 2019.

This work is licensed under a Creative Commons Attribution-NonCommercial-NoDerivatives 4.0 International (CC BY-NC-ND 4.0) License. 